\title{
TORSION OF REGIONS BOUNDED BY CIRCULAR ARCS*
}

\section{S. SOKOLNIKOFF AND E. S. SOKOLNIKOFF}

1. Introduction. N. Muschelišvili discussed recently $\nmid$ some elegant general methods of solution of a class of problems occurring in the theory of elasticity. These methods will bear closer scrutiny as the present authors have convinced themselves by obtaining, with remarkable ease, solutions of St. Venant's torsion problem for some regions bounded by circular arcs. A number of problems in harmonic and biharmonic analysis, recently discussed by other investigators, can be treated with greater simplicity by these methods.

Muschelišvili makes use of the fact that a function of a complex variable $\zeta$, analytic for $|\zeta|<1$, whose real part on $|\zeta|=1$ assumes continuous real values $u=u(\theta),(0 \leqq \theta<2 \pi)$, is given by

$$
f(\zeta)=\frac{1}{\pi i} \int_{\gamma} \frac{u(\theta) d \sigma}{\sigma-\zeta}+\text { const. }
$$

where $\sigma=e^{i \theta}$, and $\zeta$ is any point interior to the unit circle $\gamma$. It is readily shown that (1) is entirely equivalent to the formula of Schwarz.

Consider a continuous, simple closed curve $C$ in the complex $z$ plane and let $z=w(\zeta)$ map the region bounded by $C$ conformally on the unit circle $\gamma$ in the $\zeta$ plane. Let $F(z)$ be analytic in the interior of $C$, and let the value of the real part of $F(z)$ on the boundary of $C$ be $u(x, y)$.

Substituting

$$
x=\frac{w(\zeta)+\bar{w}(\bar{\zeta})}{2}, \quad y=\frac{w(\zeta)-\bar{w}(\bar{\zeta})}{2 i}
$$

in $u(x, y)$ gives

$$
u(x, y)=\phi(\sigma, \bar{\sigma})=\phi\left(\sigma, \frac{1}{\sigma}\right),
$$

where bars denote the conjugate values. Hence, from (1),

* Presented to the Society, December 30, 1937.

$\dagger$ N. Muschelišvili, Rendiconti delle Reale Accademia dei Lincei, vol. 9 (1929), pp. 295-300; Mathematische Annalen, vol. 107 (1932), pp. 282-312; Zeitschrift für Angewandte Mathematik und Mechanik, vol. 13 (1933), pp. 264-282. 


$$
F(z)=F[w(\zeta)]=\frac{1}{\pi i} \int_{\gamma} \frac{\phi\left(\sigma, \frac{1}{\sigma}\right) d \sigma}{\sigma-\zeta}+\text { const. },
$$

and the problem of Dirichlet for the region bounded by $C$ is solved.

It may be remarked that the procedure just outlined differs from that formerly used by one of the present authors* in that the integral of Poisson employed by him is replaced by a modified integral of Schwarz. The latter frequently admits of easy evaluation, in closed form, as is illustrated below in the treatment of a torsion problem.

2. Torsion problem. Consider a region $R$ of the complex $z$ plane bounded by two circular arcs $C_{1}$ and $C_{2}$ making an angle $\alpha \neq 0$ at their points of intersection $z=z_{1}$ and $z=z_{2}$. The function effecting conformal mapping of the region $R$ on the unit circle in the complex $\zeta$ plane is

$$
\zeta=\frac{C i\left(z-z_{1}\right)^{\pi / \alpha}+\left(z-z_{2}\right)^{\pi / \alpha}}{C i\left(z-z_{1}\right)^{\pi / \alpha}-\left(z-z_{2}\right)^{\pi / \alpha}} .
$$

The choice of the constants $C, z_{1}$, and $z_{2}$ is uniquely determined by the geometrical configuration and the scale used in mapping the region $R$ on the unit circle. For $\alpha=\pi / n$, where $n$ is an integer, the mapping function becomes rational, which fact greatly simplifies the evaluation of (2). To illustrate the procedure it will suffice to consider two important special cases when the region $R$ is one of the following:

(a) a lune formed by two circular arcs of equal radius and intersecting at right angles;

(b) a semi-circle.

The first of these regions suggests the profile of an airplane wing. It is easily verified that the mapping functions appropriate to the regions defined in (a) and (b) are, respectively,

$$
\zeta=\frac{2 z}{z^{2}+1}
$$

and

$$
\zeta=\frac{(z+1)^{2}-i(z-1)^{2}}{(z+1)^{2}+i(z-1)^{2}} .
$$

In obtaining (4) and (5) the constants $z_{1}$ and $z_{2}$ were chosen as -1 and +1 , which can be done without loss of generality inasmuch as

* I. S. Sokolnikoff, Transactions of this Society, vol. 33 (1931), pp. 719-732. 
this choice merely fixes the scale for the radii of arcs bounding the regions. The constant $C$ was chosen so as to make the point $z=0$ in (4) correspond to $\zeta=0$, and the point $z=0$ in (5) correspond to $\zeta=-i$.

The transformations (4) and (5) permit of easy solution, in closed forms, of the torsion problem for prisms whose cross sections are the regions defined in (a) and (b). The solution of the second of these problems is included in the papers of Greenhill, ${ }^{*}$ who attacked the problem in an entirely different way. The solution of the first problem appears to be lacking, and it will be given next.

Since the conjugate $\Psi(x, y)$ of the torsion function $\Phi(x, y)$ assumes on the boundary of the cross section of the prism the value

$$
\begin{aligned}
\Psi & =\frac{1}{2}\left(x^{2}+y^{2}\right)+\text { const. } \\
& =\frac{1}{2} z \bar{z}+\text { const. }=\frac{1}{2} w(\sigma) \bar{w}(\bar{\sigma})+\text { const. },
\end{aligned}
$$

it follows from the foregoing that the real part of the analytic function

$$
f(\zeta)=\frac{1}{2 \pi i} \int_{\gamma} \frac{w(\sigma) \bar{w}(\bar{\sigma})}{\sigma-\zeta} d \sigma+\text { const }
$$

gives the desired function $\Psi(x, y)$. From (4) it follows that $w(\sigma)$ $=\left[1-\left(1-\sigma^{2}\right)^{1 / 2}\right] / \sigma$, where the appropriate branch of the square root is determined from the observation that the imaginary part of $z$ is positive whenever $\theta$ in $\sigma=e^{i \theta}$ lies between 0 and $\pi$.

Noting that on $|\zeta|=1$ we have $\bar{\sigma}=1 / \sigma$, we deduce the relation

$$
w(\sigma) \bar{w}(\bar{\sigma})=\left[1-\left(1-\sigma^{2}\right)^{1 / 2}\right]\left[1-\left(1-\frac{1}{\sigma^{2}}\right)^{1 / 2}\right] .
$$

Substituting this expression in the integrand of (6) and evaluating the resulting elementary integrals, we obtain $\dagger$

$$
f(\zeta)=-\left(1-\zeta^{2}\right)^{1 / 2}-\frac{1-\zeta^{2}}{\pi \zeta} \log \frac{1-\zeta}{1+\zeta}+\text { const } .
$$

or if we return to the $z$-plane,

$$
F(z)=\frac{z^{2}-1}{z^{2}+1}-\frac{\left(1-z^{2}\right)^{2}}{\pi z\left(1+z^{2}\right)} \log \frac{1-z}{1+z}+\text { const } .
$$

* A. G. Greenhill, Messenger of Mathematics, vol. 8 (1879), p. 89; vol. 10 (1881), p. 83.

$\dagger$ The details of integration are of little interest and hence are omitted. In evaluating the integrals involving square roots, it is advantageous to make a substitution $\sigma=\left(1+i t^{2}\right) /\left(1-i t^{2}\right)$, so that the resulting integrals are evaluated over the real axis of the $t$ plane. 
The real part of $F(z)$, which is the desired solution, is

$$
\begin{aligned}
\Psi(x, y)= & \left\{\pi\left(x^{2}+y^{2}\right)\left[\left(x^{2}+y^{2}+1\right)^{2}-4 y^{2}\right]\right\}^{-1}\left\{\pi\left(x^{2}+y^{2}\right)\left[\left(x^{2}+y^{2}\right)^{2}-1\right]\right. \\
& +x\left(x^{2}+y^{2}+1\right)\left[4 y^{2}-\left(x^{2}+y^{2}-1\right)^{2}\right] \log R \\
& \left.+y\left(x^{2}+y^{2}-1\right)\left[\left(x^{2}+y^{2}+1\right)^{2}+4 x^{2}\right] \phi\right\},
\end{aligned}
$$

where

$$
R=\frac{\left[\left(1-x^{2}-y^{2}\right)^{2}+4 y^{2}\right]^{1 / 2}}{(1+x)^{2}+y^{2}} \text { and } \phi=\tan ^{-1} \frac{-2 y}{1-x^{2}-y^{2}} .
$$

A simple calculation shows that, on the boundary of the lune formed by $x^{2}+(y \pm 1)^{2}=2, \Psi(x, y)$ reduces to

as it should.

$$
\Psi=\frac{x^{2}+y^{2}}{2}+\text { const. }
$$

An analogous calculation* gives for the region bounded by a semicircle

$$
\begin{aligned}
f(\zeta)= & \frac{2^{1 / 2}(1+i \zeta)\left(1-\zeta^{2}\right)^{1 / 2}}{(i+\zeta)^{2}}+\frac{4}{\pi i(i+\zeta)} \\
& +\frac{2}{\pi i} \frac{\left(1-\zeta^{2}\right)}{(i+\zeta)^{2}} \log \frac{i(1-\zeta)}{1+\zeta}+\text { const. }
\end{aligned}
$$

or, if we return to the $z$-plane with the aid of (5),

$$
F(z)=\frac{1}{2 \pi i}\left\{\pi i z^{2}+\frac{2\left(z^{2}+1\right)}{z}+\frac{\left(z^{2}-1\right)^{2}}{z^{2}} \log \frac{1-z}{1+z}\right\}+\text { const. },
$$

which agrees with the result of Greenhill in the form given by Love. $\dagger$

A similar procedure can be employed in treating St. Venant's flexure problem, the solution of which, for the regions in question, does not appear in the literature. $\ddagger$

The University of Wisconsin

* The integrals appearing in this case are precisely of the same type as those occurring in the preceding problem. They are evaluated with the aid of the elementary theorems on residues, and by means of the substitution given in the preceding footnote.

$\dagger$ A. E. H. Love, A Treatise on the Mathematical Theory of Elasticity, 4th ed., 1927, p. 319.

$\ddagger$ See, however, M. Seegar and K. Pearson, Proceedings of the Royal Society, Section A, vol. 96 (1920), pp. 211-232. 